\title{
Uso de pruebas académicas individualizadas en el Politécnico Grancolombiano
}

\author{
Jaime Posada* \\ Institución Universitaria Politécnico Grancolombiano. Bogotá - Colombia
}

FECHA DE ENTREGA: 23 DE JUNIO DE 2010

FECHA DE APROBACIÓN: 29 DE ABRIL DE 2013

Resumen La asignación de pruebas académicas personalizadas, aparte de contribuir a la erradicación del fraude y el plagio, hacen posible una mejora del clima en el aula de clase. En este artículo se hace una revisión de algunas herramientas destinadas a generar múltiples cuestionarios, se describe el uso de la herramientas $\mathrm{MUCH}$, y se explica en detalle el funcionamiento del aplicativo Polirand v0.14 elaborado con el fin de mejorar algunas de las limitaciones presentes en las otras soluciones exploradas.

\begin{abstract}
The assignment of personalized academic tests, aside from contributing to the eradication of fraud and plagiarism, make possible a better classroom climate. In this article we review some tools used to generate multiple questionnaires, we describe the tool $\mathrm{MUCH}$, and explain in detail the operation of the software Polirand v0.14 developed to improve some of the limitations found in the other explored solutions.
\end{abstract}

Palabras Clave: integridad académica, cuestionarios de selección múltiple, software para la educación.

Keywords: academic integrity, multiple-choice questionnaires, software for education.

* El proyecto de investigación del cual es producto este documento, ha sido financiado por la Fundación Politécnico Grancolombiano, mediante el contrato de investigación No: 2010-DI-FICB-AMA-TC-23, aprobado el 14 de diciembre de 2009. japosada@poli.edu.co 


\section{Introducción}

La evaluación de los estudiantes puede ser una tarea difícil, especialmente para cursos con gran población. Las preguntas abiertas son quizá las más sencillas de crear, pero la calificación de ellas es tediosa, lenta y muchas veces una evaluación consistente de los estudiantes no se obtiene. De otro lado, este tipo de preguntas invitan al fraude por parte de los estudiantes, y por tanto se requiere de acciones por parte del docente que ayuden a combatirlo.

El enfoque tradicional de combatir el fraude en pruebas académicas mediante el uso de buscadores de patrones ha generado algunas herramientas cuyo funcionamiento se encuentram en [1, [5] y 6]. Dichas herramientas, bastante sofisticadas desde el punto de vista de las ciencias de la computación, son efectivas para combatir el fraude en cursos de programación donde habitualmente los códigos fuente son plagiados de internet o son circulados entre los estudiantes con pequeños cambios que intentan alterar, al menos superficialmente, la esencia del programa. Este enfoque, algo punitivo, distrae la labor docente y en su lugar, sería conveniente eliminar el fraude para no tener que combatirlo. Par lo anterior, la estrategia ideal resulta ser la asignación de pruebas individuales.

Un estudio realizado [8] ha encontrado que la asignación de pruebas individuales es muy benéfica, ya que en el caso de tareas, los estudiantes se ven obligados a discutir y entender los conceptos antes de escribir sus propias soluciones. Aunque dicho estudio contempla la enseñanza en el área de química, los beneficios no deberían diferir en otras áreas del conocimiento. La herramienta CAPA desarrollada por los autores permite generar diversos cuestionarios en forma electrónica y escrita para ser distribuidos entre los estudiantes. De forma similar al anterior estudio, en [2] se presenta el desarrollado del paquete SAIL con iguales objetivos, pero orientado a las ciencias de la computación. Entre otras herramientas similares se encuentran GTEX, GRTEX, los paquetes EXAMS, random.tex y la aplicación MUCH, que se describen en [3], [9], [10], [4] y [7] respectivamente.

Las anteriores herramientas, aunque bastante funcionales, tienen limitaciones en cuanto a:

1. Sistema operativo. CAPA, GRTEX funcionan únicamente en sistemas Unix.

2. Disponibilidad. CAPA, GRTEX, GTEX, SAIL no disponen de código fuente o ejecutables que permitan usarlos.

3. Usabilidad. RAND, GRTEX, GTEX poseen una sintaxis difícil de manejar para el usuario común.

4. Integración. Aunque todas las herramientas requieren del uso de $\mathrm{AT}_{\mathrm{E}} \mathrm{X}$ por su tipografía profesional, GTEX requiere adicionalmente del sistema GAP para álgebra discreta computacional, mientras que EXAMS requiere el uso del paquete estadístico $R$.

5. Interfaz. Ninguna de las anteriores herramientas permite un diálogo amigable y efectivo con el usuario.

En cuanto a funcionalidad global, solamente la aplicación MUCH ha logrado resultados medianamente satisfactorios en cuanto a la generación de cuestionarios 
se refiere. Por lo anterior, y con el fin de mejorar algunas de la limitaciones anteriormente expuestas, se contempló escribir la herramienta Polirand v0.14 para generar pruebas académicas en forma aleatoria, con el fin de combatir el fraude. Hace uso de la tipografía profesional de $\mathrm{AT}_{\mathrm{EX}}$ y la facilidad y conveniencia del lenguaje Python para su funcionamiento.

En la sección 2 se presenta el funcionamiento de la herramienta $\mathrm{MUCH}$, dado que esta sirvió de inspiración para la creación de Polirand v0.14. Posteriormente, en la sección 3 se muestra la forma de trabajar con Polirand v0.14 y se concluye con un análisis estadístico del impacto de este nuevo enfoque de múltiples cuestionarios frente a cursos tradicionales que hacen uso de un único temario en las pruebas académicas.

\section{Uso de MUCH}

MUCH es un programa usado para generar distintos cuestionarios de selección múltiple. Fue desarrollado por el profesor Mihalis Kolountzakis y se puede descargar en http://fourier.math.uoc.gr/ mk/much/

Cada pregunta debe escribirse en un archivo plano de texto. La primera línea corresponde al enunciado de la pregunta, mientras que las siguientes corresponden a las posibles respuestas del problema. La respuesta correcta debe ser la primera de ellas. Las líneas se separan con puntos y el archivo se finaliza también con un punto. El número máximo de respuestas es seis y no deben existir líneas en blanco o líneas que ocupen múltiples renglones. Se obtiene entonces el siguiente ejemplo de una pregunta válida:

La derivada de $\$ f(x)=x^{\wedge} 2 \$$ es:

-

$\$ f^{\prime}(x)=2 x \$$

.

$\$ f^{\prime}(x)=2 \$$

.

$\$ f^{\prime}(x)=x \$$

.

$\$ f^{\prime}(x)=0 \$$

.

Una vez se tengan todas las preguntas, los archivos se deben nombrar de forma tal que preguntas relacionadas compartan un prefijo común. Para ilustrar lo anterior, si se tienen cuatro preguntas acerca de la regla de la cadena, los archivos resultantes pueden ser cad_1.txt, cad_2.txt, cad_3.txt y cad_4.txt. De forma similar, tres preguntas acerca de derivación implícita se pueden nombrar así: imp_1.txt, imp_2.txt e imp_3.txt.

La estructura del parcial se construye al editar el archivo plano de texto test-description.tex. Aunque tiene extensión .tex, no es un archivo válido de ${ }_{A} T_{E} X$. Lo importante es la modificación de las siguientes líneas de la siguiente manera: 
seed 3701

...

serial 100;

...

use 2 from "c2_lhospitalp2_*";

use 1 from "c2_maxminp2_*";

...

create 50 ;

La primera línea inicializa el generador de números aleatorios. Aunque se puede usar cualquier número natural, se recomienda en caso de alterarlo, mantener en un lugar seguro el número usado. La segunda línea indica el primer número de serie a ser usado, esto con el fin de identificar los distintos parciales. Es posible usar otro natural. Las siguientes líneas constituyen la estructura del parcial en sí. Cada parcial tendrá dos preguntas de aquellas que comienzan con el prefijo c2_lhospitalp2_ y una pregunta de aquellas que comienzan con el prefijo c2_maxminp2_. La última línea indica el total de parciales a generar. En este caso se tendrían 50 parciales numerados desde el 100 hasta el 149. Cada parcial consta de tres preguntas.

Después de ejecutar MUCH, se obtiene el archivo mc-output.tex que contiene los múltiples cuestionarios a ser procesados por $\mathrm{LAT}_{\mathrm{E}} \mathrm{X}$. También se obtiene el importante archivo de respuestas correctas correct-answers.txt. Un ejemplo de los contenidos del archivo de respuestas es el siguiente:

$\begin{array}{llllll}\text { serial number correct answers } & \text { penalties for wrong answers } \\ 100 & \text { DEAA } & 0.250 & 0.250 & 0.250 & 0.250 \\ 101 & \text { AABA } & 0.250 & 0.250 & 0.250 & 0.250 \\ 102 & \text { EAEC } & 0.250 & 0.250 & 0.250 & 0.250 \\ 103 & \text { DECA } & 0.250 & 0.250 & 0.250 & 0.250 \\ 104 & \text { BBDD } & 0.250 & 0.250 & 0.250 & 0.250 \\ 105 & \text { CBBB } & 0.250 & 0.250 & 0.250 & 0.250\end{array}$

El anterior archivo plano de texto se puede importar fácilmente a una hoja de cálculo para facilitar el proceso de calificación de los parciales.

Aunque todo el proceso anterior representa un gran trabajo, los resultados que se obtienen son muy satisfactorios. Nada mejor que ver a los estudiantes trabajando en un ambiente que minimiza el fraude. Por otro lado, la calificación de los cuestionarios es rápida, y los resultados globales en los cursos no difieren en gran medida de cursos que usan un enfoque tradicional. Sobre lo anterior, en la sección 4 se exponen pruebas estadísticas que dan soporte a lo mismo.

\section{Uso de Polirand v0.14}

$\mathrm{Al}$ presentarse algunas limitaciones (sección 1) con otras herramientas para generación de múltiples cuestionarios, se abordó el reto de crear una herramienta que tratara de mejorar algunos de los anteriores aspectos. 
Polirand v0.14 se encuentra programado en Python, así que es sencillo generar versiones nativas para cada uno de los sistemas operativos más populares. El código fuente se encuentra liberado bajo licencia GPLv3, para que el aplicativo se pueda ofrecer a un gran número de usuarios. De otro lado, la única dependencia de Polirand v0.14 con otro software, es una instalación de $\mathrm{LAT}_{\mathrm{E}} \mathrm{X}$, lo anterior debido a su tipografía superior, y por último, el banco de problemas se encuentra unificado en un único archivo. Este banco de problemas es justamente lo que se requiere para comenzar a usar Polirand v0.14 como se expone a continuación.

\subsection{Banco de problemas}

Para que Polirand v0.14 pueda generar múltiples cuestionarios, primero se debe contar con un banco de problemas. Para tal fin, se debe iniciar con un archivo .tex con la siguiente estructura:

\documentclass [10pt] \{article\}

\usepackage [utf8] \{inputenc\}

\usepackage \{amsmath, amssymb, latexsym, amsthm\}

\usepackage [spanish] \{babel\}

\usepackage $\{$ graphicx

\usepackage [margin $=0.5 \mathrm{~cm}$, includefoot] \{geometry\}

$\backslash$ setlength $\{$ parindent\}\{0.0in\}

$\backslash$ setlength $\backslash$ parskip\}\{2pt\}

$\backslash$ makeatletter

$\backslash$ newcounter\{problemact\}

$\backslash$ stepcounter \{problemact\}

\newenvironment \{multiple\} [2]\%

$\{\{\backslash$ bf Problema SM \arabic\{problemact\}\}: \{\it Categor $\backslash$ ia: (\#1)\},\%

$\{\backslash$ it Dificultad: (\#2) $\} \backslash$ par $\}\{\backslash$ stepcounter\{problemact $\} \backslash$ par $\} \%$

$\backslash$ def $\backslash$ choice $\backslash \backslash$ ifstar \choiceok\choicestar\}

\newenvironment\{open\} [2]\%

$\{\{\backslash$ bf Problema AB \arabic\{problemact\}\}: \{\it Categor 'ia: (\#1)\},\%

$\{\backslash$ it Dificultad: (\#2) $\} \backslash$ par $\}\{\backslash$ stepcounter\{problemact $\} \backslash$ par $\} \%$

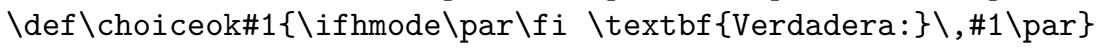

$\backslash$ def $\backslash$ choicestar\#1\{\ifhmode $\backslash p a r \backslash f i \backslash$ textbf $\{$ Falsa: $\} \backslash, \# 1 \backslash p a r\}$

$\backslash$ makeatother

\begin\{document\} }

.

\end } \{ \text { document } \}

A continuación, se pueden añadir problemas de enunciado abierto en el cuerpo del documento usando el entorno open: 


\section{$\backslash$ begin $\{$ open $\}\{\langle$ Etiqueta $\rangle\}\{\langle$ Dificultad $\rangle\}$ \\ $<$ Enunciado> \\ lend\{open\}}

donde <Etiqueta $>$ representa el nombre de la categoría a la cual pertenece el problema, $\langle$ Dificultad $>$ es un entero que representa el grado de dificultad del problema, y <Enunciado> es la pregunta como tal. Aquí es importante señalar que <Etiqueta> debe ser una cadena alfanumérica que no debe contener caracteres reservados de IAT $\mathrm{EX}$. Como ejemplo se tiene DERIVIMP, se puede usar para preguntas relacionadas con derivación implícita. También es importante mencionar que hay sensibilidad a mayúsculas y por tanto DERIVIMP no representa la misma etiqueta que derivimp. Como un ejemplo, completo se tiene el siguiente problema de razón de cambio y cómo luce después de compilado.

$\backslash$ begin open\} \{RAZONCAM $\}\{1\}$

Una cometa a $\$ 100 \$$ metros del suelo se mueve horizontalmente

con una velocidad de $\$ 8 \$ \mathrm{~m} / \mathrm{s}$. Con qul'e velocidad cambia el

\'angulo formado por la cuerda y la horizontal, cuando se

han soltado $\$ 200 \$$ metros de cuerda?

lend\{open\}

Problema AB 1: Categoría: (RAZONCAM), Dificultad: (1)

Una cometa a 100 metros del suelo se mueve horizontalmente con una velocidad de $8 \mathrm{~m} / \mathrm{s}$. Con qué velocidad cambia el ángulo formado por la cuerda y la horizontal, cuando se han soltado 200 metros de cuerda?

También es posible añadir problemas de selección múltiple con el entorno multiple:

$\backslash$ begin $\{$ multiple $\}\{\langle$ Etiqueta $\rangle\}\{\langle$ Dificultad $\rangle\}$

$<$ Enunciado>

$\backslash$ choice $*\{<$ Verdadera $\rangle\}$

$\backslash$ choice $\{<\mathrm{Falsa} 1>\}$

-

$\backslash$ choice $\{<$ Falsa $n>\}$

\end\{multiple\} }

$<$ Etiqueta $\rangle,\langle$ Dificultad $\rangle,<$ Enunciado> representan la misma información que en el entorno open, $\langle$ Verdadera $>$ corresponde a la respuesta verdadera y $<$ Falsa $1>, \ldots,<$ Falsa n> son los posibles distractores. Aunque se puede usar un número máximo de 25 , en situaciones reales es aconsejable no usar más de cinco. También hacemos notar que el lugar donde aparece la respuesta verdadera no es relevante. El comando \choice $*\{\ldots\}$ puede ir al final de los distractores, en el medio o arriba de ellos. A continuación se tiene un ejemplo completo acerca de derivación y cómo luce después de compilado. 
$\backslash$ begin $\{$ multiple $\}\{$ ALGDERIV $\}\{0\}$

La derivada de $\$ f(x)=x^{\wedge} 3 \$$ es:

lchoice $*\left\{\$ f(x)=3 x^{\wedge} 2 \$\right\}$

$\backslash$ choice $\left\{\$ f(x)=3 x^{\wedge} 3 \$\right\}$

$\backslash$ choice $\left\{\$ f(x)=x^{\wedge} 2 \$\right\}$

$\backslash$ choice $\left\{\$ f(x)=3 x^{\wedge} 4 \$\right\}$

$\backslash$ choice $\{\$ f(x)=3 x \$\}$

lend $\{$ multiple\}

Problema SM 2: Categoría: (ALGDERIV), Dificultad: (0)

La derivada de $f(x)=x^{3}$ es:

Verdadera: $f(x)=3 x^{2}$

Falsa: $f(x)=3 x^{3}$

Falsa: $f(x)=x^{2}$

Falsa: $f(x)=3 x^{4}$

Falsa: $f(x)=3 x$

Cada vez que se añada un problema, se recomienda compilarlo para verificar que no contenga errores de ningún tipo. Estos pueden ser tipográficos, propios de ${ }_{A} T_{E} X$ o de naturaleza humana como por ejemplo errar la respuesta verdadera a un problema de selección múltiple, o inclusive que ninguna de las respuestas sea la verdadera. Se aconseja ser muy metódico en esta parte, pues el control de daños puede ser complicado en una situación real que involucre estudiantes. Después de lo anterior, se puede comenzar a usar Polirand v0.14 para generar múltiples cuestionarios.

\subsection{Uso de la interfaz gráfica}

La interfaz gráfica de Polirand v0.14, la cual se ilustra en la figura 1 posee el menú "Ayuda" donde se encuentran las opciones "Instrucciones" "Plantilla de entrada" y "Licencia". En las anteriores opciones se puede acceder a instrucciones básicas de uso, una plantilla de entrada y un resumen de la licencia del programa. En menú "Archivo" se encuentran las opciones "Cargar archivo tex de entrada" y "Salir". Es precisamente en "Cargar archivo tex de entrada" donde se procede a cargar la base de datos de problemas descrita anteriormente. Después de ejecutar dicha opción, y de seleccionar el archivo indicado, se debe seleccionar las categorías activas a usar en la creación de los cuestionarios. La anterior situación se ilustra en la figura 2 A continuación se debe seleccionar el número de problemas a usar por cada una de la categorías activas como se ilustra en la figura 3

Después de completar los anteriores procedimientos, es necesario completar el formulario que se ilustra en la figura 1 Ya que la mayoría de campos del formulario se explican por sí mismos, a continuación se presentan unos comentarios sobre los campos particulares. 


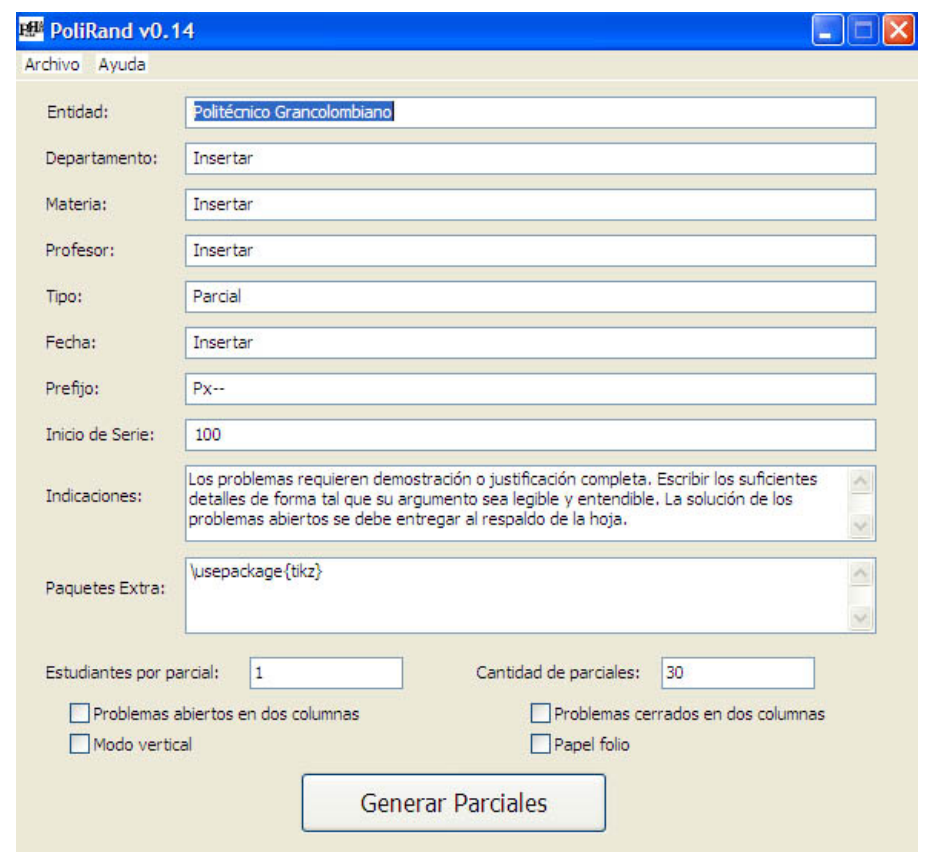

Figura 1. Interfaz de Polirand v0.14

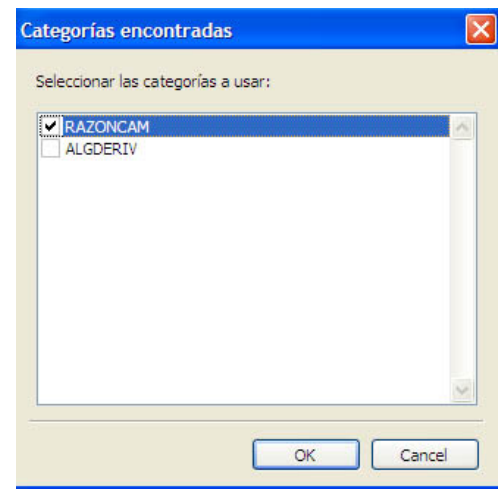

Figura 2. Selección de Categorías

Tipo. Parcial 1, Quiz 3, Taller 2, etc. Tipo de actividad a realizar.

Prefijo. Dado que cada parcial tendrá un identificador único con el fin de facilitar su calificación, el prefijo es el texto que todos comparten. Como ejemplo, un primer parcial de cálculo diferencial podría tener prefijo P1CD. 


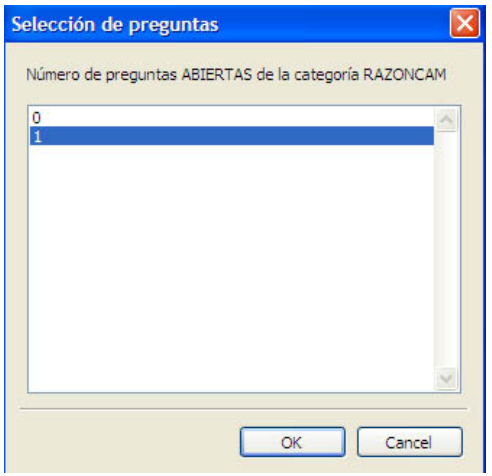

Figura 3. Número de Preguntas a Usar

Inicio de Serie. Número entero que representa el inicio de la parte variable del identificador. Si se usa el prefijo P1CD y como "Inicio de Serie" al entero 100, los parciales se identificarán como P1CD100, P1CD101, P1CD102, etc.

Indicaciones. Instrucciones y ayudas para el estudiante. Es posible escribir código de $\mathrm{LT}_{\mathrm{E}} \mathrm{X}$ directamente. A continuación se tiene un ejemplo de una entrada en dicho campo y su resultado al compilarla.

Devolver esta hoja marcada con sus datos y las respuestas en tinta a los problemas de selecci \'on $\mathrm{m} \backslash$ 'ultiple. $\backslash \backslash[1.5 \mathrm{ex}]$ $\backslash$ centerline $\backslash$ large Ayudas\}

$\backslash$ begin $\{\operatorname{multicols\} }\{2\}$

\begin } \text { enumerate\} }

$\backslash$ item $\$ \backslash$ displaystyle $\backslash f r a c\{d\}\{d x\} \backslash \tan x=\backslash \sec ^{\wedge} 2 \backslash ! x \$$

$\backslash$ item $\$ \backslash$ displaystyle $\backslash$ frac $\{\mathrm{d}\}\{\mathrm{dx}\} \backslash \sec \mathrm{x}=\backslash \sec \mathrm{x} \backslash \tan \mathrm{x} \$$

$\backslash$ item $\$ \backslash$ displaystyle $\backslash$ frac $\{\mathrm{d}\}\{\mathrm{dx}\} \backslash \csc \mathrm{x}=-\backslash \csc \mathrm{x} \backslash \cot \mathrm{x} \$$

$\backslash$ item $\$ \backslash$ displaystyle $\backslash f \operatorname{rac}\{d\}\{d x\} \backslash \cot \mathrm{x}=-\backslash \csc ^{\wedge} 2 \backslash$ ! $\mathrm{x} \$$

lend $\{$ enumerate\}

lend\{multicols\}

Indicaciones: Devolver esta hoja marcada con sus datos y las respuestas en tinta a los problemas de selección múltiple.

Ayudas
1. $\frac{d}{d x} \tan x=\sec ^{2} x$
3. $\frac{d}{d x} \csc x=-\csc x \cot x$
2. $\frac{d}{d x} \sec x=\sec x \tan x$
4. $\frac{d}{d x} \cot x=-\csc ^{2} x$ 
Paquetes Extra. En el archivo de salida de Polirand v0.14, se incluyen los siguientes paquetes (algunos con argumentos adicionales) de $\mathrm{AT}_{\mathrm{E}} \mathrm{X}$ :

\usepackage [utf8] \{inputenc\}

\usepackage \{amsmath, amssymb, latexsym, amsthm\}

\usepackage [spanish] \{babel\}

\usepackage $\{\mathrm{calc}\}$

\usepackage $\{$ graphicx $\}$

\usepackage\{multicol\}

\usepackage ifthen\}

\usepackage [margin $=0.5 \mathrm{~cm}$, includefoot] \{geometry\}

\usepackage $\{$ fancyhdr\}

Es posible adicionar paquetes extras en esta entrada del formulario. Como ejemplo, muchas veces se requieren paquetes gráficos como TikZ.

Problemas abiertos en dos columnas. Seleccionar esta opción si se desea ahorrar espacio al diagramar en dos columnas los problemas abiertos.

Problemas abiertos en dos columnas. Similar a la anterior opción. Hace lo propio con los problemas de selección múltiple.

Modo vertical. Seleccionar esta opción si se desea que las posibles respuestas a los problemas de selección múltiple aparezcan hacia abajo. En términos de $\mathrm{LT}_{\mathrm{E} X} \mathrm{X}$, las posibles respuestas se separan con el comando \newline. Es importante dejar en blanco si se desea que las posibles respuestas aparezcan hacia la derecha (separadas por el comando \quad).

Papel folio. Seleccionar esta opción si se desea papel folio de 8.5 in $\times 13$ in. Dejar en blanco si se desea papel carta de 8.5 in $\times 11$ in.

\subsection{Generación de cuestionarios}

Una vez diligenciado el formulario, se ejecuta el botón "Generar Parciales", el cual pide confirmación al usuario para crear los parciales. Aunque Polirand v0.14 propone un nombre de archivo basado en la fecha y hora en la que se crearon los parciales, dicho nombre se puede cambiar en el diálogo de la figura 4

Finalmente se debe compilar el archivo $\mathrm{AT}_{\mathrm{E}} \mathrm{X}$ de salida. Las soluciones a los problemas de selección múltiple se encuentran en la primera hoja del archivo de salida compilado. 


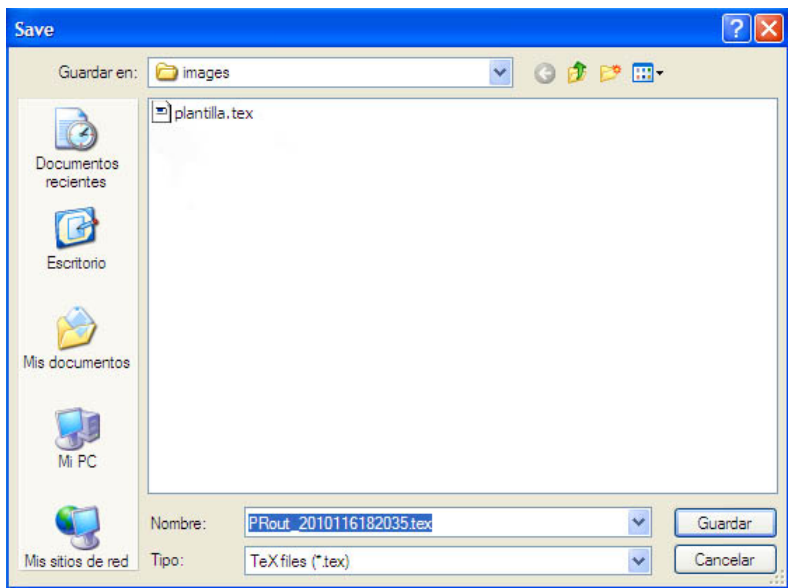

Figura 4. Guardar Archivo de Salida.

\section{Análisis estadístico}

Para analizar el impacto del uso de la metodología de cuestionarios individualizados, se realizaron pruebas de hipótesis sobre dos medias con un nivel de significancia $\alpha=0.05$. La primera muestra corresponde a la nota de 57 parciales obtenidos con MUCH en el curso de Cálculo II en el primer corte académico del semestre 2010-I, mientras que la segunda muestra corresponde a la nota de 61 parciales con enfoque tradicional. Los anteriores parciales corresponden también al primer corte académico y el profesor orientador de la materia es el mismo. En el cuadro 1 se resume la información de dichas muestras.

\begin{tabular}{rccc}
\hline & TAmaño & Media & Desviación \\
\cline { 2 - 4 } Parciales con MUCH & 57 & 2.46 & 1.12 \\
Parciales tradicionales & 61 & 2.37 & 0.9 \\
\hline
\end{tabular}

Cuadro 1. Información de las muestras. Primer corte.

Dado que $\alpha=0.05$, se obtiene que $z_{\alpha / 2}=1.96$. La hipótesis nula $H_{0}$ y la hipótesis alternativa que se usarán corresponden a:

$$
\begin{aligned}
& H_{0}: \mu_{1}=\mu_{2} \\
& H_{1}: \mu_{1} \neq \mu_{2}
\end{aligned}
$$


Al tener distintos tamaños las muestras y distintas varianzas, el estadístico de prueba que se usará corresponde a:

$$
z=\frac{\left(\bar{x}_{1}-\bar{x}_{2}\right)-d_{0}}{\sqrt{\frac{\sigma_{1}^{2}}{n_{1}}+\frac{\sigma_{2}^{2}}{n_{2}}}}
$$

donde $d_{0}=\mu_{1}-\mu_{2}$. En nuestro caso, $d_{0}=0, n_{1}=57, \bar{x}_{1}=2.46, \sigma_{1}=1.12$, $n_{2}=61, \bar{x}_{2}=2.37$ y $\sigma_{2}=0.9$. En este caso $z$ está dado por:

$$
z=\frac{(2.46-2.37)}{\sqrt{\frac{1.2544}{57}+\frac{0.81}{61}}} \approx 0.48
$$

ya que $0.48 \leq 1.96$, se tiene que $-z_{\alpha / 2} \leq z \leq z_{\alpha / 2}$ y por tanto no hay evidencia para rechazar $H_{0}$. Por tanto concluimos que la media tradicional es igual a la media al usar MUCH. De forma similar a la anterior prueba, en el cuadro 2 se detallan los resultados en el segundo corte académico del curso de Cálculo II.

\begin{tabular}{rccc}
\hline & TAmaño & Media & Desviación \\
\cline { 2 - 4 } Parciales con MUCH & 39 & 1.81 & 0.93 \\
Parciales tradicionales & 38 & 1.52 & 0.82 \\
\hline
\end{tabular}

Cuadro 2. Información de las muestras. Segundo corte.

En este caso $z$ está dado por

$$
z=\frac{(1.81-1.52)}{\sqrt{\frac{0.8649}{39}+\frac{0.6724}{38}}} \approx 1.45
$$

y dado que $1.45 \leq 1.96$, no hay evidencia para rechazar $H_{0}$. Por tanto concluimos, de nuevo, que la media tradicional es igual a la media si se usa MUCH.

\section{Conclusión}

El uso de la herramienta Polirand v0.14 permite agilizar el proceso de generar múltiples cuestionarios para pruebas académicas. Permite también facilitar el proceso de calificación de los mismos, y más importante, permite minimizar el fraude sin generar gran impacto en el desempeño global del curso. El software se encuentra liberado bajo licencia GPLv3 y se disponen de versiones nativas para cada uno de los sistemas operativos más populares. 


\section{Referencias}

1. Aiken, A.: A System for Detecting Software Plagiarism. http://theory.stanford edu/ aiken/moss/ (1994)

2. Bridgeman, S., Goodrich, M.T., Kobourov, S.G., Tamassia, R.: SAIL: A System for Generating, Archiving, and Retrieving Specialized Assignments in $\mathrm{LAT}_{\mathrm{E}} \mathrm{X}, 31 \mathrm{st}$ ACM SIGCSE Technical Symposium on Computer Science Education, 300-304. (2000)

3. Farrell, N.: GTEX: Generation of Exam Scripts and Solutions for the Faculty and Institute of Actuaries Exams. http://niallfarrell.net/NiallFarrell/ FinalYearProject_files/report.pdf (2008)

4. Fulman, I.: Randomly Generated Paper Exams. http://math.asu.edu/ ifulman/ random-exams/ (2008)

5. Gitchell, D., Tran, N.: Sim: a utility for detecting similarity in computer programs. ACM SIGCSE Bulletin. Vol. 31, no. 1, pp. 266-270. (1999)

6. Hunt, J. W., McIllroy, M.D.: An algorithm for differential file comparison. Tech. Rep. 41, Bell Labs. (1976)

7. Kolountzakis, M.: Much: a program to generate random multiple-choice questionnaires using $\mathrm{AT}_{\mathrm{E} X}$. http://fourier.math.uoc.gr/ mk/much/ (2006)

8. Morrisey, D.J., Kashy, E., Tsai, I.: Personalized assignments for freshman chemistry. Journal of Chemical Education 72, 141-146. (1995)

9. Shapiro, J.: GRTEX - To Make Multiple Exam Versions from LATEX. http://www physics.rutgers.edu/computers/grtex.pdf (1997)

10. Zeileis A., Gruen B.: Package 'exams': Automatic Generation of Standardized Exams for Large-Lecture Courses. http://cran.r-project.org/web/packages/ exams/exams.pdf (2009) 